\title{
Targeting the initial investigation and management in cases of acute pulmonary embolism
}

\author{
Nicholas Moore, Peter Hawkins
}

\begin{abstract}
It was noted by consultants in our hospital that the early investigation of suspected pulmonary embolism (PE) lacked structure. This was causing delays in definitive diagnosis and early management. The resulting unnecessary use of investigation was also wasting resources. In particular, the inappropriate use of serum d-dimer tests was causing concern. The National Institute of Clinical Excellence (NICE) guidelines recommend use of the 2-level Well's score to target investigation in suspected PE. A baseline audit against the NICE guideline revealed that Well's scores were rarely used (only calculated in $12 \%$ of cases) and confirmed the suspicion that early investigation was poorly targeted.
\end{abstract}

This project intervened using educational talks promoting the use of Well's scores in cases of suspected PE. Well's score proformas were placed in the emergency department and emergency assessment unit for reference. Their availability was advertised.

This significantly increased the use of Well's scores $(46 \%$ vs $11 \%, p<0.001)$. Fewer patients underwent unnecessary d-dimer measurements in cases of likely PE (65\% vs $86 \%)$. Initial investigation was more targeted in cases where a Well's score had been calculated than in cases without a Well's score. For example, significantly fewer unnecessary d-dimer tests were performed in these cases $(45 \%$ vs $100 \%, p<0.05)$.

The cost of unnecessary investigation in suspected PE is not only significant financially but also in the resulting delay in definitive diagnosis and management for the patient. The simple intervention used here was effective in addressing this problem.

\section{Problem}

Admitting doctors are often criticised for the inappropriate use of investigations in the early stages of diagnosis. This not only wastes resources, but it delays correct diagnosis and can in some cases be harmful to the patient.

The unnecessary measurement of d-dimer in cases of pulmonary embolism (PE) is particularly high.

\section{Background}

Symptomatic pulmonary embolism has a $>10 \%$ mortality rate in the early stages if left untreated.[1] Patients presenting with the symptom complex including pleuritic chest pain, dyspnoea, haemoptysis and evidence of venous thromboembolic disease may warrant investigation. A serum d-dimer measurement has high sensitivity and low specificity and thus is useful for ruling out PE as a cause of these symptoms if the diagnosis is unlikely. A CT pulmonary angiogram (CTPA) is both sensitive and specific and is thus ideal for definitive diagnosis of PE. CTPA is limited by a lack of resources and the high dose of radiation required for this test.

The National Institute of Clinical Excellence (NICE) guideline on venous thromboembolic diseases (published June 2012)[2] advises the use of a 2-level Well's score to determine which of these diagnostic tests is required. A low score (unlikely PE) should be investigated with d-dimer first, and CTPA in the event of a positive ddimer. A high score (likely PE) should be investigated with a CTPA as soon as possible. In either case, if a CTPA is indicated but has been delayed, an interim therapeutic dose of low molecular weight heparin (LMWH) should be given.

Close adherence to this guideline is important for quality of care for several reasons:

1. Patients with likely PE who have an inappropriate d-dimer test will delay treatment with LMWH significantly. Urgent blood tests can take more than one hour from deciding to take the sample to having the result

2. Efficiently ruling out PE in patients allows for timely investigation of alternative diagnoses

3. Needless d-dimer tests in patients with high Well's scores is a waste of resources. Likewise, any CTPA without preceding d-dimer test in cases of low likelihood PE is both a waste of resources and a large inappropriate dose of radiation.

\section{Baseline Measurement}

Baseline measurement of this problem took the form of an audit into the current adherence to the NICE guideline on diagnosis and early management of pulmonary embolism (guideline CG144). The sample included any patients admitted to the emergency admissions unit (EAU) in a 2 week period where any of the GP, the A\&E doctor, the clerking junior doctor or the medical consultant had suspected PE. Measures of adherence to the guidelines included use of 2-level Well's score, appropriate use of d-dimer in cases with a Well's score suggesting unlikely PE and inappropriate use of $d$ - 
dimer in cases with Well's score suggesting likely PE.

Our sample found that of 646 patients admitted to EAU in this 2 week period, 51 had a suspected PE. A 2-level Well's score was calculated and recorded in only 6 patients $(12 \%)$. Well's scores were calculated in retrospect to determine where investigations had been used appropriately. Fourteen of 56 patients were calculated to have a likely PE according to retrospective Well's scoring. Of these patients, $12(86 \%)$ had an inappropriate d-dimer taken prior to LMWH or CTPA investigation. In every case (12 out of 12), interim LMWH was given while waiting for CTPA.

In the case of unlikely PE, two patients underwent inappropriate diagnostic CTPA following negative d-dimer tests in this period.

Clearly there was a very low use of the 2-level Well's score in this baseline study. This appeared to be leading to a large number of inappropriate d-dimer tests and in turn delaying the early administration of LMWH. Additionally, the inappropriate use of CTPA is both a drain on resources and potentially harmful to patients.

See supplementary file: ds1831.pptx - "Baseline data"

\section{Design}

It is clear that structured adherence to guidelines and in particular the 2 level Well's score would be the key element in improving this problem.

When investigating suitable interventions with colleagues three issues became apparent:

1. Many do not use 2 level Well's scores because they either forget altogether, or because they cannot remember the components of the score.

2. The trust wide introduction of a mandatory proforma for every patient with suspected PE would be an unpopular addition to the workload of admitting doctors. This strategy would fail as a result.

3. Many doctors believed that the radiology department in our hospital would not perform a CTPA on any patient prior to having a d-dimer result.

For these reasons we selected education and access to Well's scores as the key strategies for promoting change. Short presentations were made at the hospital audit meeting and at teaching sessions for the junior doctors in accident and emergency department. These aimed to highlight the guideline and detail the results of our baseline measurements. We also discussed the Well's score and how it should be used.

\section{Strategy}

PDSA cycle 1: The use of a proforma was discussed with acute medical physicians and admitting doctors. A standardised design was available from the National Institute of Clinical Excellence website. The outcome was that an extra piece of paperwork would not be popular and would be difficult to implement for every patient.

PDSA cycle 2: The importance of using the Well's score and following NICE guidelines was discussed at multiple hospital meetings. We left Well's score proformas in A\&E and the emergency assessment unit for reference and advertised their availability. Example Well's scores were pinned to the walls in the emergency assessment unit.

These interventions were evaluated in a prospective re-audit 2 months later. Improvements between audits were determined using a $\mathrm{CHI}$ square test where $\mathrm{p}<0.05$ was considered significant.

See supplementary file: ds1834.docx - "PDSA Cycles (1)"

\section{Post-Measurement}

Data was collected over 2 weeks and used identical inclusion/exclusion criteria to the baseline study. Six hundred and ninety-seven patients were admitted during this period. Pulmonary embolus had been suspected in a total of 64 patients.

Two level Well's scores were calculated significantly more often in the post intervention measurement compared to the baseline measurement $(46 \%$ vs $11 \%, p<0.001)$.

Unnecessary tests:

D-dimer tests were performed in fewer cases of likely PE in the post intervention measurement, however this result was not statistically significant $(65 \%$ vs $86 \%, p=0.18)$. Where a Well's score had been calculated, significantly fewer d-dimers were performed for cases of likely PE $(45 \%$ vs $100 \%, p<0.05)$.

In cases of unlikely PE, only one patient underwent a CTPA following a negative $\mathrm{d}$-dimer test in the post intervention measurement $(n=44)$. This occurred in 2 out of 37 patients in the baseline measurement.

Necessary tests:

The number of CTPA's performed in cases of likely PE were similar in both the baseline and post interventional measurements $(71 \%$ for both). There were significantly more CTPA's performed in cases of likely PE where a Well's score had been calculated, compared to cases where no Well's score was used $(91 \%$ vs $33 \%, p<0.05)$.

D-dimer tests were performed in $100 \%$ of cases of unlikely PE in the post interventional study, compared to $96 \%$ in the baseline study.

In cases of unlikely PE where the d-dimer was positive the post intervention study found a higher number of patients being offered CTPA ( $55 \%$ vs $33 \%, p=0.18$ ). This figure increased to $83 \%$ where a Well's score was calculated (Well's score vs no Well's score $p$ value $=0.003$. 
See supplementary file: ds1835.pptx - "PE audit comparative data graphs"

\section{Lessons and Limitations}

The introduction of a proforma is a common way to stimulate change and improve quality. One lesson from this study was that this approach can be unpopular to the point of inhibiting the main goals of the project. The first PDSA cycle revealed that time pressured doctors were unwilling to increase their work load by filling out forms. As such it was integral to this intervention to avoid increasing paperwork for admitting doctors. Our alternative was to promote the use of a two level Well's score and advertise where to find information if required through educational reminders. This had a notable effect on clinical practice.

The most encouraging outcome measure from our project was that the use of a Well's score clearly correlated with appropriately targeted initial investigation. This provides us the confidence to continue promoting the use of Well's scores in our trust. This should also be used as evidence in other hospitals that appropriate education and use of scoring systems will translate to better adherence to national guidelines and thus improved patient care.

This study was limited by the wide differential diagnoses which can accompany suspected pulmonary embolism. The relatively low specificity of $d$-dimer tests make them positive in cases where other markers (eg clinical indications or inflammatory blood markers) point to an alternative diagnoses. In the light of other more convincing evidence a patient may not be offered a CTPA despite a positive d-dimer as it is no longer indicated. This example would be counted as contradicting the national guideline in our study. As a result any future measurements will be adjusted using pre-specified objective evidence which strongly suggests an alternative diagnosis, (for example consolidation on a chest xray in addition to raised blood inflammatory markers).

\section{Conclusion}

Investigations for suspected PE were not being used appropriately in our hospital. This was revealed in an audit which demonstrated poor adherence to national guidelines. Two level Well's scores were being used infrequently leading to unnecessary investigation in some instances, and delays to necessary investigation in other cases. In particular, the inappropriate use of d-dimer tests in cases of likely PE was causing delays in potentially live saving management.

We publicised the use of Well's scores through educational meetings and advertised where to find reference materials on the acute wards.

These measures significantly increased the use of Well's scores and improved targeting of initial investigation and management. Cases where a Well's score was used showed a significant improvement in investigation targeting compared to cases where no Well's score was performed. This confirmed that our approach of promoting the use of a scoring system was impacting on patient care. It gives us the confidence to continue with this intervention in the future.

\section{References}

1. Anderson FA, Wheeler HB, Goldberg RJ, Hosmer DW, Patwardhan NA, Jovanovic B et al. A population-based perspective of the hospital incidence and case-fatality rates of deep vein thrombosis and pulmonary embolism. Archives of Internal Medicine. 1991;151(5):933.

2. NICE guideline CG144: Venous thromboembolic diseases: the management of venous thromboembolic diseases and the role of thrombophilia testing. Issued June 2012.

\section{Declaration of interests}

None

\section{Acknowledgements}

Authors would like to acknowledge Steven Thompson for his role in the team. 